\title{
Singlet fermionic dark matter with dark $Z$
}

\author{
Dong-Won Jung ${ }^{1, \mathrm{a}}$, Soo-hyeon Nam ${ }^{1, \mathrm{~b}}$, Chaehyun Yu ${ }^{1, \mathrm{c}}$, Yeong Gyun Kim ${ }^{2, \mathrm{~d}}$, Kang Young Lee ${ }^{3, \mathrm{e}}$ \\ ${ }^{1}$ Department of Physics, Korea University, Seoul 02841, South Korea \\ 2 Department of Science Education, Gwangju National University of Education, Gwangju 61204, South Korea \\ ${ }^{3}$ Department of Physics Education \& RINS, Gyeongsang National University, Jinju 52828, South Korea
}

Received: 23 February 2020 / Accepted: 23 May 2020 / Published online: 8 June 2020

(C) The Author(s) 2020

\begin{abstract}
We present a fermionic dark matter model mediated by the hidden gauge boson. We assume the QED-like hidden sector which consists of a Dirac fermion and $\mathrm{U}(1)_{X}$ gauge symmetry, and introduce an additional scalar electroweak doublet field with the $\mathrm{U}(1)_{X}$ charge as a mediator. The hidden $\mathrm{U}(1)_{X}$ symmetry is spontaneously broken by the electroweak symmetry breaking and there exists a massive extra neutral gauge boson in this model which is the mediator between the hidden and visible sectors. Due to the $\mathrm{U}(1)_{X}$ charge, the additional scalar doublet does not couple to the Standard Model fermions, which leads to the Higgs sector of type I two Higgs doublet model. The new gauge boson couples to the Standard Model fermions with couplings proportional to those of the ordinary $Z$ boson but very suppressed, thus we call it the dark $Z$ boson. We study the phenomenology of the dark $Z$ boson and the Higgs sector, and show the hidden fermion can be the dark matter candidate.
\end{abstract}

\section{Introduction}

The Standard Model (SM) provides a consistent description of known elementary particles and interactions. The CERN Large Hadron Collider (LHC) has discovered the Higgs boson to complete the SM field contents [1,2]. Still the majority of matter in our Universe is, however, dark matter (DM) beyond the reach of our knowledge. Thus the existence of hidden sectors is an exciting possibility as an explanation of many problems beyond the SM including DM.

If $\mathrm{DM}$ is a fermion of the SM gauge singlet, a mediator field would connect DM to the SM sector with renormalizable

\footnotetext{
a e-mail: dongwonj@korea.ac.kr

be-mail: glvnsh@gmail.com

ce-mail: chyu@korea.ac.kr

de-mail: ygkim@gnue.ac.kr

e e-mail: kylee.phys@gnu.ac.kr (corresponding author)
}

couplings. One of the minimal choice for the mediator field is a real singlet scalar which is coupled to singlet fermionic dark matter (SFDM) with the Yukawa type interaction and to the SM through the quadratic term of the Higgs field, the only massive coupling in the SM lagrangian. Various aspects of such kind of minimal models, so called Higgs portal, has been studied in extensive literatures [3-20]. If there is a gauge symmetry in the hidden sector, a vector field could be the mediator between the DM field in the hidden sector and the SM fields. When the hidden gauge symmetry is $U(1)$, the corresponding gauge field can be coupled to the SM fields through the kinetic mixing with the field strength of the SM $\mathrm{U}(1)$ gauge interaction. Then the vector field has vectorlike couplings to the SM sector and is usually called a dark photon. The hidden $U(1)$ gauge symmetry is spontaneously broken in the hidden sector to yield the dark photon mass.

In this work, we consider an alternative way to connect the hidden sector including fermionic DM without the kinetic mixing to the SM. We introduce an additional scalar field which is the SM doublet and has the $\mathrm{U}(1)_{X}$ charge to connect the hidden $\mathrm{U}(1)_{X}$ gauge field to the SM fields. The new scalar doublet does not couple to the SM fermions due to the $\mathrm{U}(1)_{X}$ charge, but couples to the SM Higgs doublet in the scalar potential as well as the $\mathrm{SU}(2)_{L}$ gauge fields. Thus the Higgs sector is same as that of the two Higgs doublet model (2HDM) of type I. It is pointed out in Ref. [21] that an additional $\mathrm{U}(1)$ gauge symmetry can explain the type I 2HDM flavour structure instead of the discrete symmetry. The $\mathrm{U}(1)_{X}$ gauge boson gets the mass via the electroweak symmetry breaking (EWSB) in this model and is mixed with the $Z$ boson. Since the new gauge boson, $Z^{\prime}$, is mixed with only the $Z$ boson, its couplings to the SM fermions are same as the $Z$ boson couplings except for involving a suppression factor. Thus we call it a dark $Z$ boson. The dark $Z$ boson mass should be of the EW scale or less, and actually expected to be much light. We anticipate that the couplings of the dark $Z$ to the SM should be very small due to constraints from lots of 
low energy neutral current (NC) experiments. We consider the $\rho$-parameter, the atomic parity violation of Cs atom, and the rare decays of $K$ and $B$ mesons as experimental constraints in this work. Note that the new gauge coupling need not be extremely small to suppress the dark $Z$ couplings to the SM sector, if the Higgs doublet mixing $\sim 1 / \tan \beta$ could be small enough.

SFDM carries the $\mathrm{U}(1)_{X}$ charge and is connected to the SM through the dark $Z$ after the EWSB. Since we have no restrictions on the $\mathrm{U}(1)_{X}$ charge of SFDM, the interaction strength of SFDM $\sim g_{X} X_{\psi}$ is a new free parameter to fit the observed relic density and the DM-nucleon cross sections under the bounds from direct detection experiments of DM. We show that our SFDM mediated by the dark $Z$ can be a good DM candidate satisfying the stringent experimental constraints on the dark $Z$, DM and Higgs phenomenology.

This paper is organized as follows. We describe the model in Sect. 2. Presented are the experimental constraints on the dark $Z$ boson from the $\rho$ parameter, the atomic parity violation of $\mathrm{C}_{s}$ atom, and decays of $K$ and $B$ mesons in Sect. 3. The dark matter phenomenology is studied in Sect. 4 and the Higgs sector phenomenology in Sect. 5. We discuss the predictions for the future experiments and conclude in Sect. 6.

\section{The model}

We consider the QED-like hidden sector which consists of a SM gauge singlet Dirac fermion and the $\mathrm{U}(1)_{X}$ gauge field. No fields in the SM lagrangian carry the $\mathrm{U}(1)_{X}$ gauge charge and no kinetic mixing with the $\mathrm{SM} \mathrm{U}(1)_{Y}$ gauge field is assumed. We introduce an additional scalar field as a mediator between the hidden sector and the visible sector, which is the $\mathrm{SM} \mathrm{SU}(2)$ doublet and carries the $\mathrm{U}(1)_{X}$ charge. The charge assignment of two Higgs doublets $H_{1}$ and $\mathrm{H}_{2}$, and the hidden fermion $\psi$ based on the gauge group $\mathrm{SU}(3)_{c} \times \mathrm{SU}(2)_{L} \times \mathrm{U}(1)_{Y} \times \mathrm{U}(1)_{X}$ is given by

$H_{1}\left(1,2, \frac{1}{2}, \frac{1}{2}\right), \quad H_{2}\left(1,2, \frac{1}{2}, 0\right), \quad \psi(1,1,0, X)$,

where the $\mathrm{U}(1)_{X}$ charge of $H_{1}$ is fixed to be $1 / 2$ for convenience and that of $\psi$ is a free parameter.

Since the additional scalar doublet $H_{1}$ does not couple to the SM fermions due to the $\mathrm{U}(1)_{X}$ charge, the visible sector lagrangian of our model looks like the 2HDM of type I except for the extra $\mathrm{U}(1)_{X}$ gauge interaction for $H_{1}$. We write the Higgs sector lagrangian as

$$
\begin{aligned}
\mathcal{L}_{H}= & \left(D^{\mu} H_{1}\right)^{\dagger} D_{\mu} H_{1}+\left(D^{\mu} H_{2}\right)^{\dagger} D_{\mu} H_{2}-V\left(H_{1}, H_{2}\right) \\
& +\mathcal{L}_{\mathrm{Y}}\left(H_{2}\right),
\end{aligned}
$$

where $V\left(H_{1}, H_{2}\right)$ is the Higgs potential and $\mathcal{L}_{\mathrm{Y}}$ the Yukawa interactions of the SM fermions. The covariant derivative is defined by
$D^{\mu}=\partial^{\mu}+i g W^{\mu a} T^{a}+i g^{\prime} B^{\mu} Y+i g_{X} A_{X}^{\mu} X$,

where $X$ is the hidden $\mathrm{U}(1)_{X}$ charge operator and the $A_{X}^{\mu}$ corresponding gauge field. The Higgs potential is given by

$$
\begin{aligned}
V\left(H_{1}, H_{2}\right)= & \mu_{1}^{2} H_{1}^{\dagger} H_{1}+\mu_{2}^{2} H_{2}^{\dagger} H_{2} \\
& +\lambda_{1}\left(H_{1}^{\dagger} H_{1}\right)^{2}+\lambda_{2}\left(H_{2}^{\dagger} H_{2}\right)^{2} \\
& +\lambda_{3}\left(H_{1}^{\dagger} H_{1}\right)\left(H_{2}^{\dagger} H_{2}\right) \\
& +\lambda_{4}\left(H_{1}^{\dagger} H_{2}\right)\left(H_{2}^{\dagger} H_{1}\right) .
\end{aligned}
$$

Note that the $H_{1}^{\dagger} H_{2}$ quadratic term and the quartic term with $\lambda_{5}$ coupling are forbidden by the $\mathrm{U}(1)_{X}$ gauge symmetry.

After the EWSB, the vacuum expectation values (VEVs) of two Higgs doublets arise, $\left\langle H_{i}\right\rangle=\left(0, v_{i} / \sqrt{2}\right)^{T}$ with $i=$ 1,2 , and the gauge bosons get masses as

$$
\begin{aligned}
\mathcal{L}_{M}= & \frac{1}{4} g^{2} v^{2} W^{+} W^{-} \\
& +\frac{1}{8}\left(\begin{array}{c}
A_{X} \\
W^{3} \\
B
\end{array}\right)^{T}\left(\begin{array}{ccc}
g_{X}^{2} v_{1}^{2} & -g g_{X} v_{1}^{2} & g^{\prime} g_{X} v_{1}^{2} \\
-g g_{X} v_{1}^{2} & g^{2} v^{2} & -g g^{\prime} v^{2} \\
g^{\prime} g_{X} v_{1}^{2} & -g g^{\prime} v^{2} & g^{\prime 2} v^{2}
\end{array}\right)\left(\begin{array}{c}
A_{X} \\
W^{3} \\
B
\end{array}\right),
\end{aligned}
$$

where $v^{2}=v_{1}^{2}+v_{2}^{2}$. Diagonalizing the mass matrix with the Weinberg angle $\theta_{W}$ between $W^{3}$ and $B$, we get the massless mode, the photon, and diagonalization with the additional mixing angle $\theta_{X}$ between $A_{X}$ and the ordinary $Z$ mode follows to get the physical masses such as,

$$
\begin{aligned}
\left(\begin{array}{c}
A_{X} \\
W^{3} \\
B
\end{array}\right) & =\left(\begin{array}{ccc}
1 & 0 & 0 \\
0 & c_{W} & s_{W} \\
0 & -s_{W} & c_{W}
\end{array}\right)\left(\begin{array}{ccc}
c_{X} & s_{X} & 0 \\
-s_{X} & c_{X} & 0 \\
0 & 0 & 1
\end{array}\right)\left(\begin{array}{c}
Z^{\prime} \\
Z \\
A
\end{array}\right) \\
& =\left(\begin{array}{c}
c_{X} Z^{\prime}+s_{X} Z \\
-s_{X} c_{W} Z^{\prime}+c_{X} c_{W} Z+s_{W} A \\
s_{X} s_{W} Z^{\prime}-c_{X} s_{W} Z+c_{W} A
\end{array}\right) .
\end{aligned}
$$

where $s_{W}=\sin \theta_{W}=g^{\prime} / \sqrt{g^{2}+g^{\prime 2}}, s_{X}=\sin \theta_{X}$ and

$\tan 2 \theta_{X}=\frac{-2 g_{X} \sqrt{g^{2}+g^{\prime 2}} v_{1}^{2}}{\left(g^{2}+g^{\prime 2}\right) v^{2}-g_{X}^{2} v_{1}^{2}}=\frac{-2 g_{X} \sqrt{g^{2}+g^{\prime 2}} \cos ^{2} \beta}{\left(g^{2}+g^{\prime 2}\right)-g_{X}^{2} \cos ^{2} \beta}$,

with $\tan \beta=v_{2} / v_{1}$. Since the kinetic mixing is ignored in this work, the $Z-Z^{\prime}$ mixing $\theta_{X}$ is originated from the mixing in the mass matrix of Eq. (5). Then the neutral gauge boson masses are

$$
\begin{aligned}
m_{Z, Z^{\prime}}^{2}= & \frac{1}{8}\left(g_{X}^{2} v_{1}^{2}+\left(g^{2}+g^{\prime 2}\right) v^{2}\right. \\
& \left. \pm \sqrt{\left(g_{X}^{2} v_{1}^{2}-\left(g^{2}+g^{\prime 2}\right) v^{2}\right)^{2}+4 g_{X}^{2}\left(g^{2}+g^{\prime 2}\right) v_{1}^{4}}\right) .
\end{aligned}
$$

Note that only two mixing angles are required to diagonalize the neutral gauge boson mass matrix in this model. 
We write the NC interactions in terms of the physical states of the gauge bosons:

$$
\begin{aligned}
\mathcal{L}_{N C} \sim & -e A^{\mu} \bar{f} Q \gamma_{\mu} f-c_{X} Z^{\mu}\left(g_{L} \bar{f}_{L} \gamma_{\mu} f_{L}+g_{R} \bar{f}_{R} \gamma_{\mu} f_{R}\right) \\
& +s_{X} Z^{\prime \mu}\left(g_{L} \bar{f}_{L} \gamma_{\mu} f_{L}+g_{R} \bar{f}_{R} \gamma_{\mu} f_{R}\right),
\end{aligned}
$$

where the electric charge is defined by $Q=T_{3}+Y$ and

$$
\begin{aligned}
e & =\frac{g g^{\prime}}{\sqrt{g^{2}+g^{\prime 2}}}, \quad g_{L}=-\frac{1}{2} \frac{g^{2}-g^{\prime 2}}{\sqrt{g^{2}+g^{\prime 2}}}, \\
g_{R} & =\frac{g^{\prime 2}}{\sqrt{g^{2}+g^{\prime 2}}} .
\end{aligned}
$$

Note that $g_{L}$ and $g_{R}$ are common with $Z^{\prime}$ and $Z$ but the $Z^{\prime}$ couplings involve the suppression factor, $-\sin \theta_{X}$. This is the reason why we call $Z^{\prime}$ the dark $Z$.

The structure of the Higgs sector is almost same as that of the type I 2HDM. The only difference is that the pseudoscalar Higgs boson does not exist in this model due to being the longitudinal mode of the dark $Z$. Thus there are only three additional Higgs bosons in this model, a neutral CP-even Higgs boson and a pair of charged Higgs bosons.

The physical CP-even neutral Higgs bosons $h_{1}, h_{2}$ are defined by

$$
\begin{aligned}
\left(\begin{array}{c}
\rho_{1} \\
\rho_{2}
\end{array}\right) & =\left(\begin{array}{cc}
\cos \alpha & \sin \alpha \\
-\sin \alpha & \cos \alpha
\end{array}\right)\left(\begin{array}{l}
h_{1} \\
h_{2}
\end{array}\right) \\
& =\left(\begin{array}{c}
h_{1} \cos \alpha+h_{2} \sin \alpha \\
-h_{1} \sin \alpha+h_{2} \cos \alpha
\end{array}\right),
\end{aligned}
$$

where $\rho_{i}$ are the neutral components of the doublets, $H_{i}=$ $\left(H_{i}^{+},\left(\rho_{i}+i \eta_{i}\right) / \sqrt{2}\right)^{T}$, and the mixing angle $\alpha$ is defined by

$\tan 2 \alpha=\frac{\left(\lambda_{3}+\lambda_{4}\right) \tan \beta}{\lambda_{1}-\lambda_{2} \tan ^{2} \beta}$.

The masses are obtained by

$$
\begin{aligned}
M_{1,2}^{2}= & \lambda_{1} v_{1}^{2}+\lambda_{2} v_{2}^{2} \\
& \mp \sqrt{\left(\lambda_{1} v_{1}^{2}-\lambda_{2} v_{2}^{2}\right)^{2}+\left(\lambda_{3}+\lambda_{4}\right)^{2} v_{1}^{2} v_{2}^{2}} .
\end{aligned}
$$

The heavier mode $h_{2}$ is the SM Higgs and $h_{1}$ is the extra neutral Higgs boson with relevant values of parameters as will be shown later.

The charged Higgs boson masses are diagonalized to get the physical mode $H^{ \pm}$by,

$$
\begin{aligned}
\left(\begin{array}{l}
H_{1}^{ \pm} \\
H_{2}^{ \pm}
\end{array}\right) & =\left(\begin{array}{cc}
\cos \beta & \sin \beta \\
-\sin \beta & \cos \beta
\end{array}\right)\left(\begin{array}{c}
G^{ \pm} \\
H^{ \pm}
\end{array}\right) \\
& =\left(\begin{array}{c}
G^{ \pm} \cos \beta+H^{ \pm} \sin \beta \\
-G^{ \pm} \sin \beta+H^{ \pm} \cos \beta
\end{array}\right),
\end{aligned}
$$

where the mixing angle is $\beta$ in this case. One of the diagonalized masses is given by

$m_{ \pm}^{2}=-\frac{1}{2} \lambda_{4}\left(v_{1}^{2}+v_{2}^{2}\right)=-\frac{1}{2} \lambda_{4} v^{2}$, for $H^{ \pm}$and the other is 0 for $G^{ \pm}$. The massless mode $G^{ \pm}$ is the Goldstone mode eaten up to be the longitudinal mode of the $W^{ \pm}$boson. We write the Yukawa interactions for the charged Higgs boson with the short-hand notation

$$
\begin{aligned}
\mathcal{L}_{Y}= & -g_{i j}^{d} \bar{Q}_{L}^{i} H_{2} d_{R}^{j}-g_{i j}^{u} \bar{Q}_{L}^{i} \tilde{H}_{2} u_{R}^{j}-g_{i j}^{l} \bar{L}_{L}^{i} H_{2} l_{R}^{j}+H . C ., \\
= & -\frac{\sqrt{2} \cot \beta}{v} H^{+}\left(m_{d} \bar{u}_{L} V_{\mathrm{CKM}} d_{R}\right. \\
& \left.-m_{u} \bar{u}_{L} V_{\mathrm{CKM}} d_{R}-m_{l} \bar{v}_{L} l_{R}^{-}\right)+H . C .
\end{aligned}
$$

where $V_{\mathrm{CKM}}$ are the corresponding quark mixings.

\section{Dark $Z$ phenomenology}

The NC interactions with the dark $Z$ boson are constrained by various experiments. Apart from the new Higgs masses and mixings, the independent model parameters are $\left(g_{X}, \tan \beta\right)$ in our model lagrangian. Instead in this analysis, we present the results in terms of the observables $\left(m_{Z^{\prime}},-s_{X}\right)$.

\subsection{The $\rho$ parameter}

We consider the precision test on the electroweak sector using the $\rho$ parameter. The $\rho$ parameter is defined by the ratio of $W$ and $Z$ boson masses, $\rho \equiv m_{W}^{2} / m_{Z}^{2} c_{W}^{2}$, and should be 1 at tree level in the SM. In this model, we have $m_{W}=g v / 2$ as in the SM at tree level. But the $Z$ boson mass is shifted such that

$m_{Z}^{2}=\frac{m_{W}^{2}}{c_{W}^{2} c_{X}^{2}}-m_{Z^{\prime}}^{2} \frac{s_{X}^{2}}{c_{X}^{2}}$,

and then the inverse of the $\rho$ parameter is

$$
\frac{1}{\rho}=\frac{m_{Z}^{2} c_{W}^{2}}{m_{W}^{2}}=\frac{1}{c_{X}^{2}}-\frac{m_{Z^{\prime}}^{2} c_{W}^{2}}{m_{W}^{2}} \frac{s_{X}^{2}}{c_{X}^{2}} \approx 1+s_{X}^{2}\left(1-\frac{m_{Z^{\prime}}^{2} c_{W}^{2}}{m_{W}^{2}}\right),
$$

in the leading order of $s_{X}^{2}$. The deviation $\Delta \rho$ from the unity is defined by

$\rho \equiv \frac{1}{1-\Delta \rho}$,

then the leading contribution to $\Delta \rho$ in this model is given by

$\Delta \rho_{X}=-s_{X}^{2}\left(1-\frac{m_{Z^{\prime}}^{2} c_{W}^{2}}{m_{W}^{2}}\right)$.

The correction $\Delta \rho$ is related to $T$ parameter as [22]

$\Delta \rho=\alpha\left(m_{Z}\right) T$

of which values are

$T=0.07 \pm 0.12$, 
Fig. 1 Excluded regions in $\left(M_{Z^{\prime}},-s_{X}\right)$ plane. The green (light grey) region is excluded by too small $\Delta \rho$ and the blue (dark grey) region excluded by too large $\Delta \rho$. The pink (grey) region shows exclusions by rare $K$ and $B$ decays. The solid line denotes the atomic parity violation bound and the region above the line is excluded. The yellow region is disfavoured by the nonperturbative $\mathrm{U}(1)_{X}$ gauge coupling, $g_{X}>4 \pi$. The allowed points of $\left(M_{Z^{\prime}},-s_{X}\right)$ fall on the white triangular region

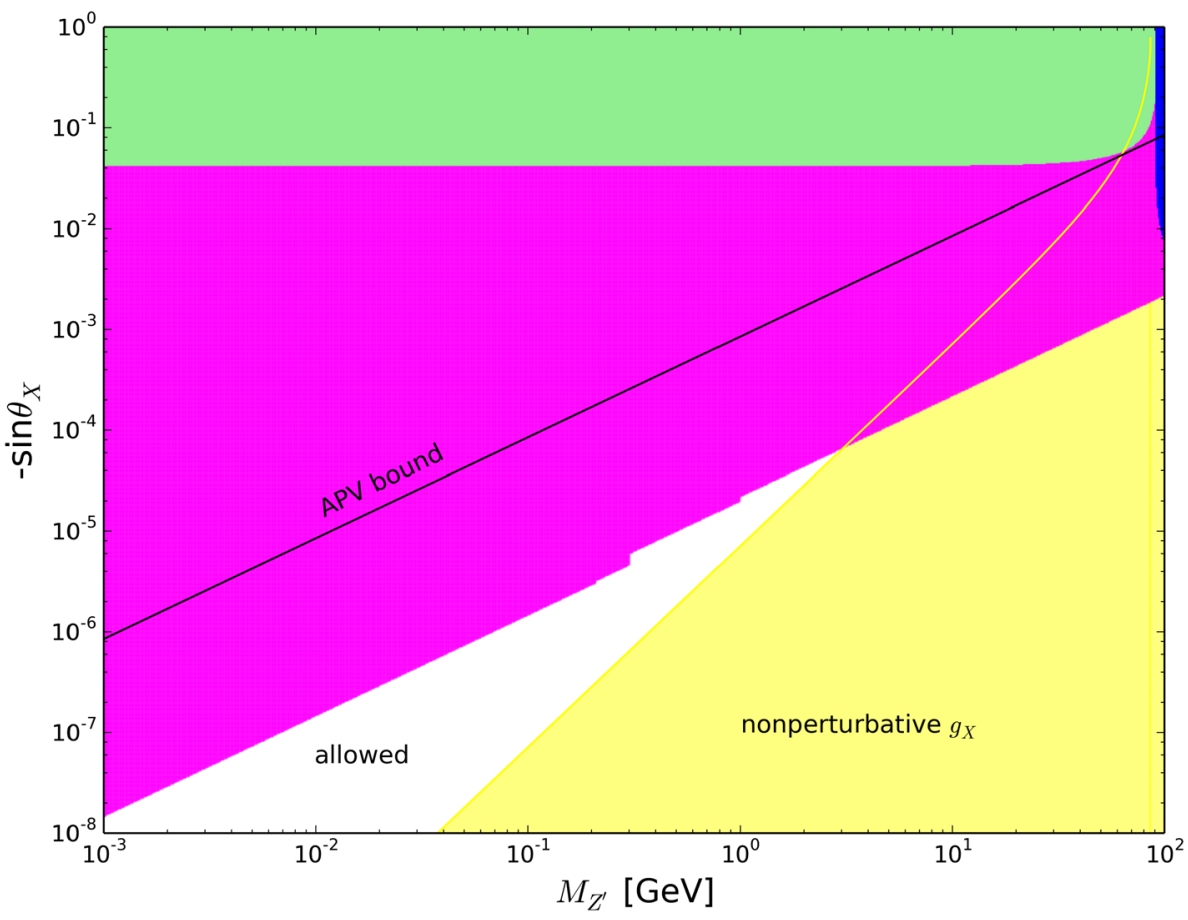

and $\alpha^{(5)^{-1}}\left(m_{Z}\right)=127.955 \pm 0.010$ obtained in Ref. [33]. Then we have bounds for $\Delta \rho$ as

$-0.00039<\Delta \rho<0.001485$.

Applying this bound to $\Delta \rho_{X}$, we show the excluded regions in $\left(m_{Z^{\prime}},-s_{X}\right)$ plane in Fig. 1. The green (light grey) region denotes too small $\Delta \rho$ and the blue (dark grey) region too large $\Delta \rho$. Note that the yellow region denotes the breakdown of the perturbativity, $g_{X}>4 \pi$.

\subsection{The atomic parity violation}

The parity violation of the atomic spectra is observed due to the $Z$ boson exchanges. The precise measurement of the atomic parity violation (APV) provides a strong constraint on the exotic NC interactions. We derive the effective lagrangian for the corresponding process as

$$
-\mathcal{L}=-\frac{G_{F}}{\sqrt{2}}\left(g_{A V}^{u}\left(\bar{e} \gamma_{\mu} \gamma^{5} e\right)\left(\bar{u} \gamma^{\mu} u\right)+g_{A V}^{d}\left(\bar{e} \gamma_{\mu} \gamma^{5} e\right)\left(\bar{d} \gamma^{\mu} d\right)\right),
$$

at the quark level.

The APV is described by the weak charge of the nuclei defined by

$Q_{W} \equiv-2\left[Z g_{A V}^{p}+N g_{A V}^{n}\right]$,

where $Z(N)$ is the number of protons (neutrons) in the atom and the nucleon couplings are defined by $g_{A V}^{p} \equiv 2 g_{A V}^{u}+g_{A V}^{d}$ and $g_{A V}^{n} \equiv g_{A V}^{u}+2 g_{A V}^{d}$. In the SM, $g_{A V}^{p} \approx-1 / 2+2 s_{W}^{2}$ and $g_{A V}^{n} \approx 1 / 2$ lead to $Q_{W}^{S M} \approx-N+Z\left(1-4 s_{W}^{2}\right)$ at tree level, which is shifted by the dark $Z$ contribution as

$Q_{W}=Q_{W}^{S M}\left(1+\frac{m_{Z}^{2}}{m_{Z^{\prime}}^{2}} s_{X}^{2}\right)$,

in the leading order of $s_{X}$. The SM prediction of the Cs atom is [23-27]

$Q_{W}^{S M}=-73.16 \pm 0.05$,

and the present experimental value is $[28,29]$

$Q_{W}^{\text {exp }}=-73.16 \pm 0.35$

which yields the bound

$\frac{m_{Z}^{2}}{m_{Z^{\prime}}^{2}} s_{X}^{2} \leq 0.006$

at $90 \%$ CL [30-32]. This constraint is shown as the solid line of the $\left(m_{Z^{\prime}},-s_{X}\right)$ plane in Fig. 1. The region above the line is excluded.

\subsection{Rare meson decays}

The flavour physics have been a good laboratory of new physics. Davoudiasl et al. [30-32] suggest that the flavourchanging neutral current (FCNC) decays of $K$ and $B$ mesons provide strong constraints on the dark $Z$ model. Here, we follow their analysis to constrain our model. 
The FCNC interactions of the dark $Z$ boson $s \rightarrow d Z^{\prime}$ and $b \rightarrow s Z^{\prime}$ derive $K \rightarrow \pi Z^{\prime}$ and $B \rightarrow K\left(K^{*}\right) Z^{\prime}$ decays,

$$
\begin{aligned}
\operatorname{Br}\left(K^{+} \rightarrow \pi^{+} Z^{\prime}\right) & \approx 4 \times 10^{-4}\left(\frac{m_{Z}}{m_{Z^{\prime}}}\right)^{2} s_{X}^{2}, \\
\operatorname{Br}\left(B \rightarrow K Z^{\prime}\right) & \approx 0.1\left(\frac{m_{Z}}{m_{Z^{\prime}}}\right)^{2} s_{X}^{2},
\end{aligned}
$$

and sequential decays of $Z^{\prime}$ into lepton pairs lead to rare decays $K \rightarrow \pi l \bar{l}$ and $B \rightarrow K l \bar{l}$. The experimental measurements for $K$ mesons

$$
\begin{aligned}
\operatorname{Br}\left(K^{+} \rightarrow \pi^{+} e^{+} e^{-}\right) & =(3.00 \pm 0.09) \times 10^{-7} \\
\operatorname{Br}\left(K^{+} \rightarrow \pi^{+} \mu^{+} \mu^{-}\right) & =(9.4 \pm 0.6) \times 10^{-8} \\
\operatorname{Br}\left(K^{+} \rightarrow \pi^{+} v^{+} v^{-}\right) & =(1.7 \pm 1.1) \times 10^{-10}
\end{aligned}
$$

and for $B$ mesons

$$
\begin{aligned}
\operatorname{Br}\left(B \rightarrow K l^{+} l^{-}\right) & =(4.51 \pm 0.23) \times 10^{-7}, \\
\operatorname{Br}\left(B^{+} \rightarrow K^{+} \nu \nu\right) & <1.6 \times 10^{-5},
\end{aligned}
$$

are obtained [33]. Then the strongest constraints are derived [30-32]

$$
\begin{aligned}
& \left|\frac{m_{Z}}{m_{Z^{\prime}}} s_{X}\right| \leq \frac{0.001}{\sqrt{\mathrm{Br}\left(Z^{\prime} \rightarrow l^{+} l^{-}\right)}}, \\
& \left|\frac{m_{Z}}{m_{Z^{\prime}}} s_{X}\right| \leq \frac{0.001}{\sqrt{\mathrm{Br}\left(Z^{\prime} \rightarrow \text { missing }\right)}} .
\end{aligned}
$$

Although being not manifest in the analysis, the DM mass affects these constraints. If the DM mass is less than the half of the $Z^{\prime}$ mass, the DM pair production channel opens and even dominates the decay rates, $\operatorname{Br}\left(Z^{\prime} \rightarrow\right.$ missing $) \sim 100 \%$ due to the $s_{X}^{2}$ suppression of the $Z^{\prime}$ decays into the SM final states. Then the $\operatorname{Br}\left(K^{+} \rightarrow \pi^{+} l^{+} l^{-}\right)$and $\operatorname{Br}\left(B \rightarrow K l^{+} l^{-}\right)$ constraints do not work. The pink (grey) region in Fig. 1 denotes the excluded points by the constraints given in Eq. (33).

The final result is depicted in Fig. 1, where constraints from $\Delta \rho, \mathrm{APV}$, and rare meson decays are presented altogether. We find that the rare meson decays provide the strongest constraints on $m_{Z^{\prime}}$ and $\left|s_{X}\right|$. We also see that the dark $Z$ is rather light, $m_{Z^{\prime}} \leq 2 \mathrm{GeV}$, and the coupling should be very small due to the small mixing angle $\left|\sin \theta_{X}\right| \leq 4 \times 10^{-5}$ as expected.

We note that the sign of $\theta_{X}$ is not determined by the phenomenological study of this section. At this stage, we just know that only very small $\left|\theta_{X}\right|$ are allowed by the experiments. Then we can see that $g_{X}$ or $\cos \beta$ should be small in the Eq. (7) for $\left|\theta_{X}\right|$ to be very small, $<10^{-5}$ and then $\theta_{X}$ should be negative. As we see that the allowed region of Fig. 1 is near the nonperturbativity region of $g_{X}$, actually only the small $\cos \beta$ (means very large $\tan \beta$ ) is allowed by our analysis.

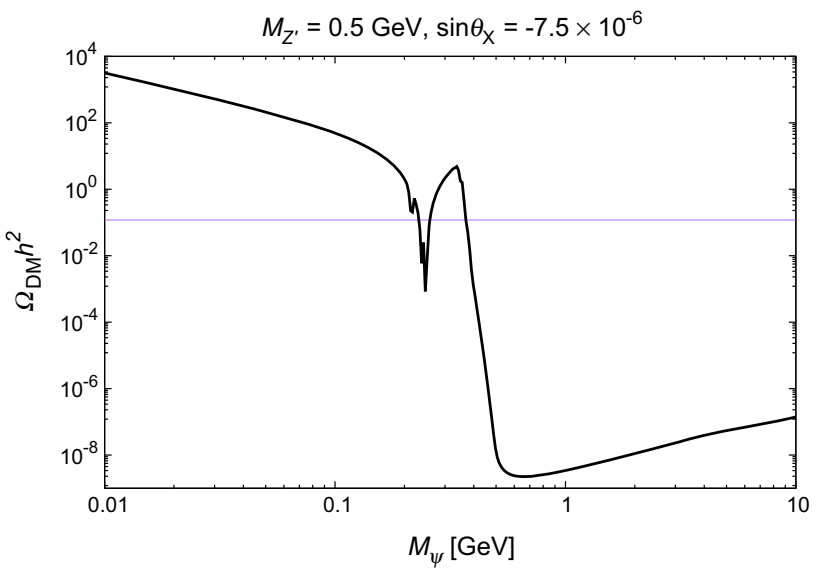

Fig. 2 The relic density with respect to the DM mass for the benchmarking point $\left(m_{Z^{\prime}}, \sin \theta_{X}\right)=\left(0.5 \mathrm{GeV},-7.5 \times 10^{-6}\right)$

\section{Dark matter phenomenology}

Our hidden sector consists of a Dirac fermion with a $\mathrm{U}(1)_{X}$ gauge symmetry. The hidden sector lagrangian is QED-like

$\mathcal{L}_{\mathrm{hs}}=-\frac{1}{4} F_{X}^{\mu \nu} F_{X \mu \nu}+\bar{\psi} i \gamma^{\mu} D_{\mu} \psi-M_{\psi} \bar{\psi} \psi$,

where

$D^{\mu}=\partial^{\mu}+i g_{X} A_{X}^{\mu} X$

and $X$ is the $\mathrm{U}(1)_{X}$ charge operator for $\psi$. We show that the singlet fermion $\psi$ can be a DM candidate. Using Eq. (6), we find that $\psi$ has vectorial interactions with $Z$ and $Z^{\prime}$ bosons,

$\mathcal{L}_{\mathrm{DM}}^{i n t}=i g_{X} X \bar{\psi} \gamma^{\mu} \psi\left(c_{X} Z^{\prime}{ }_{\mu}+s_{X} Z_{\mu}\right)$.

We have two additional parameters, $m_{\psi}$ and the $\mathrm{U}(1)_{X}$ charge for the DM phenomenology. Since the couplings between the DM sector and the SM sector involves a suppression factor $\left|s_{X}\right|$, the collider phenomenology including $\mathrm{DM}$ is affected very little with the $s_{X}^{2}$ suppression.

The SFDM contribution to the relic abundance density $\Omega$ is obtained from global fits of various cosmological observations. We can read the present value of $\Omega$ of the cold nonbaryonic DM as

$\Omega_{\mathrm{CDM}} h^{2}=0.1186 \pm 0.0020$,

from measurements of the anisotropy of the cosmic microwave background (CMB) and of the spatial distribution of galaxies [33]. Such precise value provides a stringent constraint on the model parameters. We calculate $\Omega$ and the DMnucleon cross section using the micrOMEGAs [34] with the allowed values of parameters $\left(m_{Z^{\prime}}, \sin \theta_{X}\right)$ given in the previous section. Figure 2 shows the relic density with respect to the DM mass for the benchmarking point $m_{Z^{\prime}}=0.5 \mathrm{GeV}$ and $\sin \theta_{X}=-7.5 \times 10^{-6}$. The acceptable DM annihilations for the relic abundance arise at the resonant region where $M_{\psi} \sim$ 


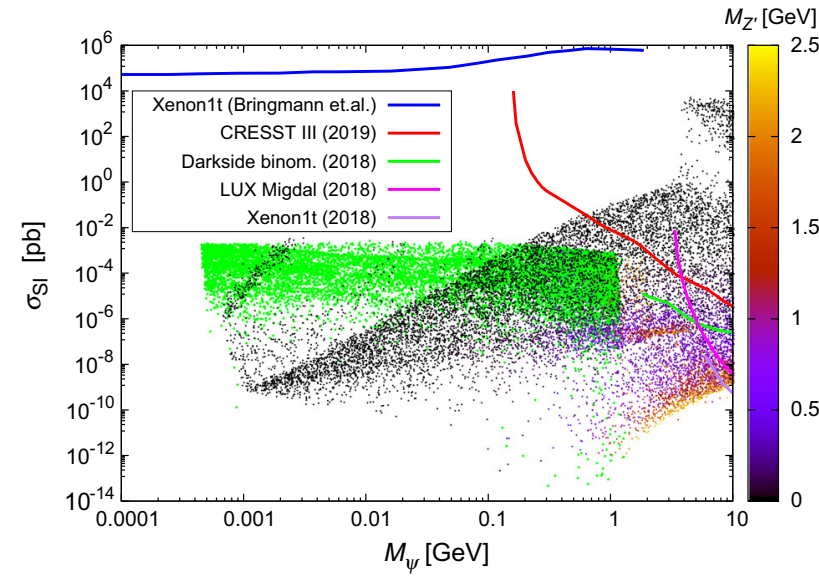

Fig. 3 The DM-nucleon cross sections for the parameter sets satisfying the relic abundance. Green points denote the resonant region for DM annihilation and points of other colors the nonresonant annihilations depending on the $Z^{\prime}$ and $h_{1}$ masses

$m_{Z^{\prime}} / 2$ through the $s$-channel $\psi \bar{\psi} \rightarrow Z^{\prime} \rightarrow$ SM particles and at the nonresonant region through the $t$-channel $\psi \bar{\psi} \rightarrow Z^{\prime} Z^{\prime}$ and the Higgsstrahlung $\psi \bar{\psi} \rightarrow Z^{\prime} h_{1}$ processes.

The direct detection cross sections for SFDM are calculated for the parameters satisfying the relic abundance of Eq. (37) and shown with respect to the DM mass in Fig. 3. We can see two groups of allowed points in the plot. The green points denote the resonant annihilations, hence $M_{\psi} \sim m_{Z^{\prime}} / 2$ and are distributed in the region of $M_{\psi}<1.2 \mathrm{GeV}$. Points of other colors for the nonresonant annihilations are distributed in the whole region of $M_{\psi}$, but are excluded by the present experiments when $M_{\psi}>10 \mathrm{GeV}$. The experimental bounds from Xenon1t [35], CRESST III [36], Darkside [37], and LUX [38] are shown together.

We have to mention that DM also interacts with the SM through the ordinary $Z$ boson. For the contributions to the relic density and the DM-nucleon cross sections, the suppression factor for coupling strengths are same order for both $Z$ and $Z^{\prime}$ mediation. Thus the dark $Z$ mediation is dominant when the DM mass is around the dark $Z$ mass, and the ordinary $Z$ mediation dominant when the DM mass around $m_{Z}$. However, the $Z$ mediated contributions are excluded by the stringent experimental bound when the DM mass is a few tens $\mathrm{GeV}$.

Production of energetic particles due to self-annihilation of DM in high DM density regions like galactic center has been studied by several telescopes as the indirect signal of DM. The present observations provide constraints on the velocity-weighted annihilation cross sections for various channels, but among them, the $\tau^{+} \tau^{-}$or $b \bar{b}$ channels are strongly constrained by energetic photon spectrum data. We take a benchmarking point with values $M_{\psi} \approx 2.1 \mathrm{GeV}$, $m_{Z^{\prime}} \approx 0.15 \mathrm{GeV},-\sin \theta_{X} \approx 1.1 \times 10^{-6}$, which satisfies the observed relic abundance and gives the DM-nucleon cross section of order $10^{-6} \mathrm{pb}$. Since this DM fermion is rather light, the $\tau^{+} \tau^{-}$channel is more relevant. The annihilation rate for the $\tau^{+} \tau^{-}$channel is $\langle\sigma v\rangle \approx 1.8 \times 10^{-37} \mathrm{~cm}^{3} \mathrm{~s}^{-1}$ much below the observed bounds $\sim 10^{-27} \mathrm{~cm}^{3} \mathrm{~s}^{-1}[39,40]$. We survey the DM mass, $2<M_{\psi}<3$ in $\mathrm{GeV}$, and find that the annihilation rate is generically small, $\langle\sigma v\rangle<10^{-34}$ $\mathrm{cm}^{3} \mathrm{~s}^{-1}$ due to the small mixing $\left|s_{X}\right|$. Thus our model is safe for the present bounds from the indirect search of DM.

\section{Higgs phenomenology}

An additional Higgs doublet that is chraged under the $\mathrm{U}(1)_{X}$ symmetry is introduced and extra scalar particles exist in our model. This set-up is similar to the Type-I 2HDM with $\mathrm{U}(1)_{H}$ gauge symmetry in Ref. [41]. We note that in both models the quartic term with the $\lambda_{5}$ coupling is forbidden due to additional gauge symmetry. However, by introducing another singlet field $\Phi$, the latter is allowed to have the $H_{1}^{\dagger} H_{2}$ quadratic term which can make extra scalars relatively heavy, whereas, in our model, the masses of extra scalar bosons are bounded by the perturbativity condition, for example, $m_{ \pm}^{2}=$ $-\frac{1}{2} \lambda_{4} v^{2} \lesssim(616 \mathrm{GeV})^{2}$. In our model the CP-odd scalar mode is eaten up by the dark $Z$ boson and there exists no CPodd scalar. This is the noticeable difference from the particle contents in the ordinary $2 \mathrm{HDM}$ as well as in the 2HDM with $\mathrm{U}(1)_{H}$ gauge symmetry [41]. As a result the new particles are an neutral Higgs boson and a pair of charged Higgs bosons. Most of the phenomenology of the Higgs sector is governed by quartic couplings of the Higgs potential. Hence we just discuss two issues on the Higgs phenomenology, the charged Higgs search and the Higgs invisible decays here.

To begin with we investigate the scalar masses. The masses are calculated with the perturbativity conditions on the quartic couplings $\left|\lambda_{i}\right|<4 \pi$ and the vacuum stability conditions [41],

$\lambda_{1}>0, \quad \lambda_{2}>0, \quad \lambda_{3}>-2 \sqrt{\lambda_{1} \lambda_{2}}, \quad \lambda_{3}+\lambda_{4}>-2 \sqrt{\lambda_{1} \lambda_{2}}$.

We find that $h_{1}$ is very light in this model since $v_{1} \ll v_{2}$. Hence $h_{2}$ should be the SM Higgs boson. If we fix the mass of $h_{2}$ to be $125.18 \pm 0.16 \mathrm{GeV}$, the $h_{1}$ mass is less than $1.2 \mathrm{GeV}$. The charged Higgs boson mass is determined by $\lambda_{4}$ solely in this model and has the upper bound $\sim 616 \mathrm{GeV}$ due to the perturbativity bound of $\lambda_{4}$. We note that these features are very insensitive to the parameter set allowed in the previous analysis.

For the analysis of $\Delta \rho$ in the previous section, we consider only the dark $Z$ contributions. By the way, the additional Higgs bosons also contribute to the $\rho$ parameter such as [42]

$\Delta \rho_{\mathrm{NS}}^{(1)}=\frac{\alpha}{16 \pi m_{W}^{2} s_{W}^{2}}\left(m_{ \pm}^{2}-\frac{m_{1}^{2} m_{ \pm}^{2}}{m_{1}^{2}-m_{ \pm}^{2}} \log \frac{m_{1}^{2}}{m_{ \pm}^{2}}\right)$, 
where $m_{1}$ is the mass of $h_{1}$ and $m_{ \pm}$the charged Higgs boson mass. Since $h_{1}$ is very light compared with $H^{ \pm}, \Delta \rho_{\mathrm{NS}}^{(1)}$ crucially depends only on the charged Higgs mass. If $m_{ \pm} \geq 120$ $\mathrm{GeV}, \Delta \rho_{\mathrm{NS}}^{(1)}$ exceeds 0.001485 of the experimental upper limit given in Eq. (23) and no parameter set can satisfy the $\Delta \rho$. On the other hand, $\Delta \rho_{\mathrm{NS}}^{(1)}$ is very sensitive to $m_{ \pm}$and it does not play a role of constraints if $m_{ \pm}$is just slightly smaller than $120 \mathrm{GeV}$, e.g. $119 \mathrm{GeV}$. (The cyan region of Fig. 1 is overlapped by other constraints.) Therefore we demand $80 \mathrm{GeV}<m_{ \pm}<120 \mathrm{GeV}$ in this model. The modelindependent lower bound is given in [33].

The recent CMS data for $H^{ \pm} \rightarrow \tau^{ \pm} v$ and $H^{+} \rightarrow t \bar{b}$ channels at $\sqrt{s}=13 \mathrm{TeV}$ with an integrated luminosity of $35.9 \mathrm{fb}^{-1}$ have been reported in Refs. [43,44]. Sanyal [45] provides the analysis of the CMS data and shows the allowed values of $\tan \beta$ and the charged Higgs mass $m_{ \pm}$for several versions of the $2 \mathrm{HDM}$ including type I. Since our charged Higgs is rather light, $m_{ \pm}<120 \mathrm{GeV}$, it is produced from the top decay and decays into $\tau v$ and $W^{ \pm} h_{1}$. Figures 3 and 5 in Ref. [45] depict the exclusion regions of $\left(m_{ \pm}, \tan \beta\right)$ in the 2HDM of type I from the upper limits of the CMS observations $\sigma_{H^{ \pm}} \operatorname{Br}\left(H^{ \pm} \rightarrow \tau^{ \pm} \nu\right)$ and $\operatorname{Br}\left(B \rightarrow X_{s} \gamma\right.$ constraints. We find the conservative limit is $\tan \beta>15$ for any values of $m_{ \pm}$from these plots. Meanwhile the parameter sets in the allowed region given in Fig. 1 correspond to very large $\tan \beta$, numerically $\tan \beta>500$ for all $m_{ \pm}$, much larger than the CMS conservative limit. Therefore the present LHC bound for $H^{ \pm}$is not relevant to our model.

Since the dark $Z$ boson is light in this model, the Higgs boson decays into the dark $Z$ pair are possible which contributes to the Higgs invisible decay modes. However, the $h_{2} Z^{\prime} Z^{\prime}$ coupling is suppressed by $\sin ^{2} \theta_{X}$ or $g_{X}^{2} \cos \beta \sin \alpha$. Since $\sin \theta_{X} \sim g_{X} \cos ^{2} \beta$ and $\sin \alpha \sim \cos \beta$, the decay rate $\Gamma\left(h \rightarrow Z^{\prime} Z^{\prime}\right)$ is suppressed by the factor $\sin ^{2} \theta_{X}$ or less compared with $\Gamma(h \rightarrow Z Z)$. Thus the $h \rightarrow Z^{\prime} Z^{\prime}$ contribution to the Higgs invisible decay is much smaller than the current limit $\operatorname{Br}(h \rightarrow$ invisible $)<0.22$ by the CMS $[46,47]$.

\section{Concluding remarks}

We have constructed the SFDM model mediated by the dark $Z$ boson. The hidden $\mathrm{U}(1)$ gauge boson does not couple to the SM sector directly in this model, but interacts with the SM through the Higgs mixing with an additional Higgs doublet involving the hidden $U(1)$ charge. The Higgs mixing induces the $Z-Z^{\prime}$ mixing, and the mixing angle depends upon the Higgs mixing angle $\beta$ and the hidden gauge coupling $g_{X}$. The dark $Z$ boson is severely constrained by the electroweak data and thus the $Z-Z^{\prime}$ mixing angle $\theta_{X}$ should be very small, $\left|\sin \theta_{X}\right|<4 \times 10^{-5}$. The allowed parameter space by the experiments is corresponding to the very large $\tan \beta$ region. The mass of the dark $Z$ is approximately the VEV of the additional Higgs doublet $v_{1}=v \cos \beta$ and consequently it is rather light, less than $2 \mathrm{GeV}$. Such a dark $Z$ boson could also affect the precision QED tests. With values of the allowed $\left(m_{Z^{\prime}},-\sin \theta_{X}\right)$ points in our analysis, additional contributions of the dark $Z$ to the anomalous magnetic moments of the electron and the muon are negligible compared with the limit given in Ref. [51].

In this model, our DM is a SM singlet fermion and mediated by the dark $Z$ boson. We find that it can satisfy the observed relic abundance from the CMB observation. Since the dominant channels of the DM annihilation in the early universe are the $s$-channel at the dark $Z$ resonance region, the $t$-channel at the $\psi \bar{\psi} \rightarrow Z^{\prime} Z^{\prime}$ opening region, and the Higgsstrahlung into $Z^{\prime} h_{1}$ region, the DM mass is same order as the dark $Z$ mass, $\sim \mathrm{GeV}$ and less.

The dark $Z$ boson might live long if the DM mass is larger than the half of the dark $Z$ mass, (actually in that case, the DM mass is almost same as the dark $Z$ mass to satisfy the relic density.) since the coupling strength of the dark $Z$ to the $\mathrm{SM}$ matter is very small. Then the proposed intensity frontier experiments, e.g. SHiP [48], FASER [49], MATHUSLA [50] and etc. will have the chance to probe the dark $Z$ boson directly in the future.

Acknowledgements This work is supported by Basic Science Research Program through the National Research Foundation of Korea (NRF) funded by the Ministry of Science, ICT, and Future Planning under the Grants No. NRF-2017R1E1A1A01074699 (DWJ, ShN), and No. NRF2017R1A2B4011946 (CY), No. NRF-2020R1A2C3009918 (CY), and also funded by the Ministry of Education under the Grants No. NRF2018R1D1A1B07047812 (DWJ), No. NRF-2018R1D1A1B07050701 (YGK), and No. NRF-2018R1D1A3B07050649 (KYL).

Data Availability Statement This manuscript has associated data in a data repository. [Authors' comment: All data included in this manuscript are available upon request by contacting with the corresponding author.]

Open Access This article is licensed under a Creative Commons Attribution 4.0 International License, which permits use, sharing, adaptation, distribution and reproduction in any medium or format, as long as you give appropriate credit to the original author(s) and the source, provide a link to the Creative Commons licence, and indicate if changes were made. The images or other third party material in this article are included in the article's Creative Commons licence, unless indicated otherwise in a credit line to the material. If material is not included in the article's Creative Commons licence and your intended use is not permitted by statutory regulation or exceeds the permitted use, you will need to obtain permission directly from the copyright holder. To view a copy of this licence, visit http://creativecomm ons.org/licenses/by/4.0/.

Funded by $\mathrm{SCOAP}^{3}$.

\section{References}

1. G. Aad et al., ATLAS collaboration. Phys. Lett. B 716, 1 (2012)

2. S. Chatrchyan et al., CMS Collaboration, Phys. Lett. B 716, 30 (2012)

3. B. Patt, F. Wilczek, arXiv:hep-ph/0605188 
4. Y.G. Kim, K.Y. Lee, Phys. Rev. D 75, 115012 (2007)

5. M. Pospelov, A. Ritz, M.B. Voloshin, Phys. Lett. B 662, 53 (2008)

6. Y.G. Kim, K.Y. Lee, S. Shin, JHEP 05, 100 (2008)

7. Y.G. Kim, S. Shin, JHEP 05, 036 (2009)

8. L. Lopez-Honorez, T. Schwetz, J. Zupan, Phys. Lett. B 716, 179 (2012)

9. M. Fairbairn, R. Hogan, JHEP 1309, 022 (2013)

10. S. Esch, M. Klasen, C.E. Yaguna, Phys. Rev. D 88, 075017 (2013)

11. M.A. Fedderke, J.-Y. Chen, E.W. Kolb, L.-T. Wang, JHEP 1408, $122(2014)$

12. K. Ghorbani, JCAP 1501, 015 (2015)

13. Y.G. Kim, K.Y. Lee, C.B. Park, S. Shin, Phys. Rev. D 93, 075023 (2016)

14. Y.G. Kim, C.B. Park, S. Shin, JHEP 12, 036 (2018)

15. M. Ettefaghi, R. Moazzemi, Eur. Phys. J. C 77, 343 (2017)

16. C.-F. Chang, X.G. He, J. Tandean, JHEP 1704, 107 (2017)

17. E. Hardy, JHEP 1806, 043 (2018)

18. Y.G. Kim, K.Y. Lee, S.-H. Nam, Phys. Lett. B 782, 316 (2018)

19. Y.G. Kim, K.Y. Lee, S.-H. Nam, Phys. Rev. D 100(7), 075038 (2019)

20. A. Filimonova, S. Westhoff, JHEP 1902, 140 (2019)

21. P. Ko, Y. Omura, C. Yu, Phys. Lett. B 717, 202 (2012)

22. M.E. Peskin, T. Takeuchi, Phys. Rev. D 46, 381 (1992)

23. W.J. Marciano, A. Sirlin, Phys. Rev. D 27, 552 (1983)

24. W.J. Marciano, A. Sirlin, Phys. Rev. D 29, 75 (1984)

25. W.J. Marciano, A. Sirlin, Phys. Rev. D 31, 213(E) (1985)

26. W.J. Marciano, J.L. Rosner, Phys. Rev. Lett. 65, 2963 (1990)

27. W.J. Marciano, J.L. Rosner, Phys. Rev. Lett. 68, 898(E) (1992)

28. S.G. Porsev, K. Beloy, A. Derevianko, Phys. Rev. D 82, 036008 (2010)

29. S.G. Porsev, K. Beloy, A. Derevianko, Phys. Rev. Lett. 102, 181601 (2009)

30. H. Davoudiasl, H.S. Lee, W.J. Marciano, Phys. Rev. D 85, 115019 (2012)

31. H. Davoudiasl, H.S. Lee, W.J. Marciano, Phys. Rev. Lett. 109, $031802(2012)$
32. H. Davoudiasl, H.S. Lee, W.J. Marciano, Phys. Rev. D 89, 095006 (2014)

33. M. Tanabashi et al., Particle Data Group, Phys. Rev. D 98(3), 030001 (2018)

34. G. Blanger, F. Boudjema, A. Goudelis, A. Pukhov, B. Zaldivar, Comput. Phys. Comm. 231, 173 (2018)

35. T. Bringmann, M. Pospelov, Phys. Rev. Lett. 122(17), 171801 (2019)

36. A.H. Abdelhameed et al., CRESST Collaboration, Phys. Rev. D 100(10), 102002 (2019)

37. P. Agnes et al., DarkSide Collaboration, Phys. Rev. Lett. 121(8), 081307 (2018)

38. D.S. Akerib et al., LUX Collaboration, Phys. Rev. Lett. 122(13), 131301 (2019)

39. M. Ackermann et al., The Fermi-LAT Collaboration, Phys. Rev. Lett. 115, 231301 (2015)

40. H. Abdallah et al., H.E.S.S. Collaboration, Phys. Rev. Lett. 117, 111301 (2016)

41. P. Ko, Y. Omura, C. Yu, JHEP 1401, 016 (2014)

42. S. Hessesberger, W. Hollik, Eur. Phys. J. C 77, 178 (2017)

43. A.M. Sirunyan et al., CMS Collaboration, JHEP 1907, 142 (2019)

44. A.M. Sirunyan et al., CMS Collaboration, arXiv:1908.09206 [hepex]

45. P. Sanyal, Eur. Phys. J. C 79, 913 (2019)

46. V. Khachatryan et al., CMS collaboration. JHEP 1702, 135 (2017)

47. A.M. Sirunyan et al., CMS Collaboration, Phys. Lett. B 793, 520 (2019)

48. S. Alekhin et al., SHiP Collaboration, Rep. Prog. Phys. 79(12), 124201 (2016)

49. A. Ariga et al., FASER Collaboration, arXiv:1812.09139

50. C. Alpigiani et al., MATHUSLA Collaboration, arXiv:1811.00927

51. M. Pospelov, Phys. Rev. D 80, 095002 (2009) 